Published in final edited form as:

ACS Appl Mater Interfaces. 2017 April 05; 9(13): 11440-11450. doi:10.1021/acsami.6b16854.

\title{
Design of Insulin-Loaded Nanoparticles Enabled by Multi-Step Control of Nanoprecipitation and Zinc Chelation
}

\author{
Sunandini Chopra ${ }^{1}$, Nicolas Bertrand ${ }^{2,3}$, Jong-Min Lim ${ }^{1,4,5,6}$, Amy Wang ${ }^{4}$, Omid C. \\ Farokhzad $5,7,8,{ }^{*}$, and Rohit Karnik ${ }^{1,{ }^{*}}$ \\ ${ }^{1}$ Department of Mechanical Engineering, Massachusetts Institute of Technology, 77 \\ Massachusetts Avenue, Cambridge, MA 02139 USA \\ ${ }^{2}$ Faculty of Pharmacy, Laval University, CHU de Quebec Research Center, 2705 Laurier Blvd., \\ Quebec City, QC, G1V 4G2 Canada \\ ${ }^{3}$ David H. Koch Institute for Integrative Cancer Research, Massachusetts Institute of Technology, \\ 500 Main Street, Cambridge, MA 02139 USA \\ ${ }^{4}$ Department of Chemical Engineering, Massachusetts Institute of Technology, 77 Massachusetts \\ Avenue, Cambridge, MA 02139 USA \\ ${ }^{5}$ Center for Nanomedicine, Brigham and Women's Hospital, 60 Fenwood Road, Boston, MA \\ 02115 USA \\ ${ }^{6}$ Department of Chemical Engineering, Soonchunhyang University, 22 Soonchunhyang-ro, \\ Shinchang-myeon, Asan-si, Chungcheongnam-do 31538, Korea \\ ${ }^{7}$ Department of Anesthesiology, Brigham and Women's Hospital, Harvard Medical School, 75 \\ Francis Street, Boston, MA 02115 USA \\ ${ }^{8}$ King Abdulaziz University, Jeddah, Saudi Arabia
}

\begin{abstract}
Nanoparticle (NP) carriers provide new opportunities for controlled delivery of drugs, and have potential to address challenges such as effective oral delivery of insulin. However, due to the difficulty of efficiently loading insulin and other proteins inside polymeric NPs, their use has been mostly restricted to the encapsulation of small molecules. To better understand the processes involved in encapsulation of proteins in NPs, we study how buffer conditions, ionic chelation, and preparation methods influence insulin loading in poly (lactic-co-glycolic acid)-b-poly(ethylene glycol) (PLGA-PEG) NPs. We report that, although insulin is weakly bound and easily released from the NPs in the presence of buffer ions, insulin loading can be increased by over 10-fold with the use of chelating zinc ions and by the optimization of the $\mathrm{pH}$ during nanoprecipitation. We further provide ways of changing synthesis parameters to control NP size while maintaining high insulin loading. These results provide a simple method to enhance insulin loading of PLGA-PEG
\end{abstract}

*Correspondence: karnik@mit.edu, ofarokhzad@bwh.harvard.edu.

Notes: O.C.F. discloses financial interest in Selecta Biosciences and Tarveda Therapeutics.

Supporting Information: Details about measurement of insulin loading, in vitro insulin release, zeta potential, nanoparticle stability during storage and conformational stability of insulin is available in the supplementary information. 
NPs, and provide insights that may extend to other protein drug delivery systems that are subject to limited loading.

\section{Graphical abstract}

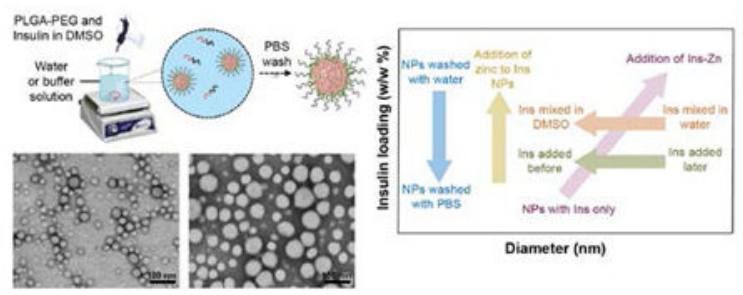

\section{Keywords}

PLGA-PEG nanoparticles; zinc; oral drug delivery; diabetes; biologics; nanomedicine; insulin

\section{Introduction}

Recent years have seen the development of many protein-based therapeutics that, due to their biological nature, are expected to be less toxic and more successful in clinical trials than their chemically synthesized counterparts ${ }^{1}$. These therapeutic proteins include monoclonal antibodies, cytokines, tissue growth factors, vaccines, and gene transfer products $^{2}$ that are used for prevention and treatment of diseases, e.g. biologic diseasemodifying anti-rheumatic drugs ${ }^{3}$, monoclonal antibodies for cancer $^{4}$, and insulin for diabetes ${ }^{5}$. However, therapeutic proteins tend to have poor chemical and physical stability against external factors like $\mathrm{pH}$, temperature, and surface interactions. The high molecular weight of proteins, their ionizable nature, their susceptibility to gastrointestinal enzymes, and their inability to permeate the mucosal tissue and the epithelial barrier in the intestine, all limit their oral bioavailability ${ }^{6}$. Administration of most biologics is therefore limited to parenteral route, which is more invasive and not favored by patients.

Among these therapeutics, oral delivery of insulin - a hydrophilic protein that plays a key role in regulating glucose metabolism - is particularly important due to the high global incidence of diabetes ${ }^{7}$. To improve the compliance of patients and decrease the number of subcutaneous injections required to control diabetes, oral, nasal, pulmonary and ocular routes of administration have been proposed for insulin ${ }^{8-9}$. In fact, an inhaled formulation of insulin was approved by the FDA in 2006, but was discontinued in 2007 due to increased costs and device design complications ${ }^{8}$. Regardless, oral delivery is regarded as the most patient-compliant route of administering insulin.

Recently, polymeric nanoparticle (NP) carriers have shown promise for drug delivery for treatment of a variety of diseases ${ }^{10-18}$, including oral delivery of proteins and of insulin for type II diabetes ${ }^{6,19-23}$. Relevant to oral delivery, NPs can be easily prepared from a variety of materials, targeting ligands on NPs can facilitate therapeutics to cross the epithelial barrier $^{21}$, and NPs can protect encapsulated and adsorbed therapeutics from $\mathrm{pH}$ changes and enzymatic degradation in the gastrointestinal $\operatorname{tract}^{21,24-25}$. Some proteins that have been 
incorporated in polymeric NP-based drug delivery systems include tetanus toxoid ${ }^{26}$, insulin $^{27}$, and interleukin $2(\mathrm{IL}-2)^{28}$.

NPs prepared from a variety of materials such as chitosan, gelatin, poly-alkyl-cyanoacrylates, dextran and polycaprolactone have been explored for oral insulin delivery ${ }^{19-21,29}$. However, PLGA-PEG NPs and poly (lactic acid)- $b$-poly (ethylene glycol) (PLA-PEG) NPs are advantageous in that they have been extensively studied as protein delivery carriers because of their biodegradability and biocompatibility, well-established safety in clinical trials, tunable surface properties for targeted delivery, and their capability to protect the protein drug from $\mathrm{pH}$ and proteolytic enzymes ${ }^{22,30-31}$. Previous studies have also shown gastric stability of PLGA-PEG, which makes it an ideal material for oral delivery platforms ${ }^{21,32-33}$. In particular, PLA-PEG NPs designed to exploit the FcRn transcytosis pathway to cross the gastroepithelial barrier and enter into the bloodstream have shown remarkable promise for oral delivery of insulin ${ }^{6}$. The FcRn transport pathway involves targeting insulin-loaded NPs to the neonatal Fc receptor ( $\mathrm{FcRn}$ ) that mediates active transport of IgG antibodies across the epithelial barrier in the GI tract, resulting in efficient transport across the intestinal epithelium into the systemic circulation for insulin delivery. However, utilizing the FcRn pathway requires small NP size; both in vitro studies using Caco-2 cells (human adenocarcinoma cell line) and in vivo studies in rats have shown that the uptake of smaller NPs $(\sim 100 \mathrm{~nm})$ is significantly more efficient than that of larger NPs $(500 \mathrm{~nm}, 1 \mu \mathrm{m}, 5 \mu \mathrm{m})^{24}$. However, it has been challenging to achieve high loading of hydrophilic protein drugs, including insulin, in PLGA-PEG or PLA-PEG NPs while maintaining small NP size, because the hydrophobic core (i.e., PLGA and PLA) resists interactions with the hydrophilic protein drugs ${ }^{34}$. For example, the insulin loading in the NPs used in the FcRn study was low $(\sim 0.5 \mathrm{wt} \%)^{6}$; assuming $10 \%$ bioavailability and typical insulin dose of $\sim 3 \mathrm{mg}$ per dosage ${ }^{35}$, a $0.5 \mathrm{wt} \%$ loading translates to an impractically high oral intake of $\sim 6 \mathrm{~g} \mathrm{NP} /$ dosage. On the other hand, although some studies have shown high insulin loading in particles ${ }^{33,36-37}$, the particle size $(17.4 \mu \mathrm{m}-120 \mathrm{~nm})$ is too large to permit effective use of transcytosis pathways to cross the epithelial barrier of the intestine. Hence, better understanding of the factors and mechanisms that determine insulin loading, with the goal of designing NPs with higher insulin loading while retaining a small NP size, is essential.

In this work, we study the role of key process steps and conditions in the synthesis of insulin-loaded PLGA-PEG NPs (Ins-NPs) formed by nanoprecipitation, which enables modification of the NP synthesis process to realize enhanced insulin loading while maintaining sub-100 nm NP size ${ }^{38}$. Specifically, we demonstrate the synthesis of insulinzinc-PLGA-PEG NPs (Ins-Zn-NPs) that uses chelating zinc ions to achieve a greater than 10 -fold increase in insulin loading while maintaining small size $(<100 \mathrm{~nm})$ required for FcRn-mediated transcytosis ${ }^{6}$. We also show that these NPs are stable for long-term storage and that insulin maintains it conformational structure after being loaded in the NPs. This study provides insights into the mechanisms of formation of Ins-NPs and illustrates simple methods for improving insulin loading in PLGA-PEG NPs while controlling NP size. This approach can extend to similar polymeric and protein systems and can lead to significant advancements in the area of NPs based protein delivery by proving a systematic way of overcoming the two main challenges i.e., improving protein loading and controlling NP size. 


\section{Experimental Section}

\subsection{Materials}

Human recombinant insulin, dimethyl sulfoxide (DMSO), and zinc chloride were purchased from Sigma-Aldrich. PLGA $10 \mathrm{k}-\mathrm{PEG}_{5 \mathrm{k}}$ was purchased from Akina Inc. (West Lafayette, IN, USA). Micro bicinchoninic acid (BCA) protein assay kit was purchased from Lamda Biotech, Inc. (St. Louis, MO, USA). Phosphate Buffer Saline (PBS) was purchased from Life Technologies (Green Island, NY, USA). Deionized (DI) water was used for all the experiments.

\subsection{Methods}

2.2.1. Synthesis of Ins-NPs and Ins-Zn-NPs-For the preparation of Ins-NPs by the nanoprecipitation method (Figure 1A), we premixed $120 \mu \mathrm{L}$ of insulin $(10 \mathrm{mg} / \mathrm{mL})$ solution in DMSO with $400 \mu \mathrm{L}$ of $\mathrm{PLGA}_{10 \mathrm{k}}-\mathrm{PEG}_{5 \mathrm{k}}(10 \mathrm{mg} / \mathrm{mL})$ solution in DMSO. The resulting solution was added drop wise to $4 \mathrm{~mL}$ DI water or buffer solution. The solution was stirred for at least two hours at $2000 \mathrm{rpm}$. The resulting NP solution was purified by ultrafiltration using Amicon Ultracel $100 \mathrm{kDa}$ membrane filters to remove free insulin and organic solvent. The NPs were washed twice with PBS and twice with DI water at $3000 \mathrm{~g}$ centrifugation for 15 min each or 4 times with DI water only. The NPs were resuspended in PBS to a final concentration of $10 \mathrm{mg} / \mathrm{mL}$. As controls, NPs were prepared with PLGA-PEG solution alone using the same procedure without the addition of insulin. In all experiments, the ratio of the organic to the aqueous phase remained constant at 13:100.

Ins-Zn-NPs were prepared by first mixing insulin $(10 \mathrm{mg} / \mathrm{mL})$ and zinc chloride $(5 \mathrm{mg} / \mathrm{mL})$ in DMSO in 1:3, 1:6, and 1:9 molar ratios of insulin:zinc. To $120 \mu \mathrm{L}$ of this solution, $400 \mu \mathrm{L}$ of $\mathrm{PLGA}_{10 \mathrm{k}}-\mathrm{PEG}_{5 \mathrm{k}}(10 \mathrm{mg} / \mathrm{mL})$ was added. The resulting solution was added drop wise to $4 \mathrm{~mL}$ DI water or buffer solution for $2 \mathrm{~h}$ under magnetic stirring at $2000 \mathrm{rpm}$. The NPs were purified by the aforementioned process.

2.2.2. Characterization of Ins-NPs and Ins-Zn-NPs-The amount of insulin loaded in Ins-NPs and Ins-Zn-NPs was quantified by using a low protein BCA assay kit. After the purification process, Ins-NPs and Ins-Zn-NPs were resuspended in PBS to a final concentration of $1 \mathrm{mg} / \mathrm{mL}$ and heated at $60^{\circ} \mathrm{C}$ for $1 \mathrm{~h}$ to release insulin. Then, $125 \mu \mathrm{L}$ of NP solution was added to each well in a 96 well-plate followed by the addition of $125 \mu \mathrm{L}$ of bicinchoninic acid (BCA) assay working solution. After incubating the NPs at $60^{\circ} \mathrm{C}$ for 30 min, the amount of insulin was analyzed using a TECAN UV spectrophotometer (Mannedorf, Switzerland) according to the manufacturer's instructions. As a control, NPs without insulin were also measured by the same procedure. The weight percent of insulin in the NPs was measured as the insulin loading and the fraction of insulin in the NPs was measured as encapsulation efficiency. Three separate experiments were performed for each data point (Supporting Information. Details of the measurement of insulin loading).

For measuring the hydrodynamic radius and zeta potential, the NPs were washed and reconstituted in $1 \mathrm{~mL}$ of DI water at a NP concentration of $0.5 \mathrm{mg} / \mathrm{mL}$. The solution was loaded in disposable low-volume cuvettes to measure the hydrodynamic radius and NP size 
distribution and in folded capillary cells to measure the zeta potential using the Zetasizer NanoZS instrument (Malvern Instruments Ltd., U.K.).

2.2.3. In vitro insulin release-The in vitro release of insulin was studied by dividing a batch of Ins-Zn-NPs equally into $100 \mathrm{kDa}$ Float A Lyzer (Spectrum Labs) units and incubating them at $37^{\circ} \mathrm{C}$ in $\mathrm{PBS}$ ( $\mathrm{pH} 7.4$ ). At each predetermined time interval, an aliquot of the NPs was taken and measured for insulin loading using the BCA assay, which included heating at $60^{\circ} \mathrm{C}$ for $1 \mathrm{~h}$ as previously described. In order to increase the accuracy of the dialysis setup, a $3 \mathrm{~mm}$ magnetic stirrer was inserted in each Float A Lyzer unit, to allow continuous mixing of the Ins-Zn-NP solution inside the dialysis chambers. Furthermore, the beaker with the dialysis chambers was kept on an orbital shaker, to ensure mixing of the buffer solution outside the dialysis chambers. Three experiments were performed for each data point (Supporting Information. Details of the measurement of in vitro release of insulin from NPs).

2.2.4. Transmission Electron Microscopy-Ins-NPs and PLGA-PEG NPs without insulin were imaged using a FEI Tecnai TEM at an acceleration voltage of $80 \mathrm{kV}$. The TEM sample was prepared by depositing $10 \mu \mathrm{L}$ of the NP suspension $(1.0 \mathrm{mg} / \mathrm{mL})$ onto a 200 mesh formvar-carbon-coated copper grid. After 20 min of incubation, excess sample on the grid was blotted away with filter paper. The grids were negatively stained for another $20 \mathrm{~min}$ at room temperature with sterile-filtered $2 \%(\mathrm{w} / \mathrm{v})$ uranyl acetate aqueous solution. The grids were then washed once with DI water and air-dried prior to imaging. The TEM images of Ins-NPs and PLGA-PEG NPs without insulin are shown in Figure 1B and 1C.

TEM-based energy-dispersive X-ray spectroscopy (EDX) was performed using a JEOL-2100 Field Emission Electron Microscope at $200 \mathrm{kV}$ with an Oxford X-Max $80 \mathrm{~mm}^{2}$ high angle annular dark field (HAA DF) detector. The NP samples were prepared on a 200mesh carbon coated TEM grid. $7 \mu \mathrm{L}$ of the sample was deposited on each grid and was allowed to completely dry out. The grids were then washed once with DI water and air-dried prior to the EDX analysis.

\section{Results and Discussion}

Nanoprecipitation, oil-in-water (O/W) emulsification-solvent evaporation, and water-in-oilin-water (W/O/W) emulsification-solvent evaporation are the most commonly used methods for making PLGA-PEG NPs. Nanoprecipitation (also called solvent displacement method) is a simple method that produces smaller NPs as compared to other methods ${ }^{17,39}$. However, some of its drawbacks include poor loading of hydrophilic drugs ${ }^{40}$ and incomplete removal of the organic solvent after NP formation ${ }^{41}$. On the other hand, emulsification-solvent evaporation methods $(\mathrm{O} / \mathrm{W}$ and $\mathrm{W} / \mathrm{O} / \mathrm{W})$ enable better loading of hydrophobic and hydrophilic drugs and allow better removal of the organic solvent ${ }^{41}$, but the size of the NPs generally ranges from a few hundred nanometers to a few hundred micrometers ${ }^{42}$ that is too large for effective use with transcytosis pathways such as the FcRn pathway ${ }^{6,24}$. We therefore focused on nanoprecipitation and leveraged the fact that it can enable production of smaller NPs as compared to the other commonly used synthesis methods of double and 
single emulsion, while investigating ways of overcoming its drawback of poor insulin loading.

Ins-NPs were prepared by nanoprecipitation by first dissolving PLGA-PEG and insulin in an organic solvent (DMSO), which is miscible in the aqueous phase ${ }^{43}$, and then adding the solution drop-wise to a stirred aqueous solution (water or buffer solution) to form NPs (Figure 1A). Here, DMSO is the solvent and water is the anti-solvent. Once in contact with water, PLGA-PEG and insulin self-assemble to form spherical NPs. TEM images in Figure $1 \mathrm{~B}$ and $1 \mathrm{C}$ show that Ins-NPs and empty PLGA-PEG NPs are spherical and fairly monodisperse with diameters less than $100 \mathrm{~nm}$.

The long-term stability of NPs was accessed by measuring the size and zeta potential of the NPs before and after freeze-drying them; an insignificant change in particle size and zeta potential was observed (Supporting Information. Measurement of particle size and zeta potential before and after undergoing the process of freeze-drying (Figure S5a and b)). The stability of the NPs in solution was also studied and it revealed that particle size was stable up to 4 days in water after which an increase in particle size was observed potentially due to aggregation (Supporting Information. Measurement of particle size up to 4 days in water (Figure S5c).

The conformational integrity of insulin after encapsulation in freshly synthesized NPs as well as NPs that were stored after freeze-drying was confirmed using circular dichroism spectroscopy (Supporting Information. Measurement of preserved secondary structure of insulin after encapsulation and release from NPs using circular dichroism (Figure S5d)). Lopes et al. reported that the released insulin retained its secondary structure as indicated by the $\mathrm{CD}$ data ${ }^{44}$. The same insulin was also biologically active, which was analyzed in diabetic rats. Another study where the preservation of the secondary structure of insulin as evident from the $\mathrm{CD}$ data also exhibited retained bioactivity in vivo when analyzed used steptozotocin (STZ)-induced diabetic rats ${ }^{45}$. Lastly, in another study the CD data indicated the presence of conformational integrity, which mapped to confirmed bioactivity as analyzed by a cell-based protein kinase B (AKT) phosphorylation assay as well as an STZ-induced animal model. These examples suggest that the preservation of the secondary structure of insulin as indicated by $\mathrm{CD}$ is indicative of preserved potential biologically activity of the $\operatorname{protein}^{46}$.

\subsection{Effect of washing buffer on insulin loading}

Several studies have reported insulin loading in polymeric NPs after washing the NPs with water only ${ }^{47-49}$. However, since water wash alone is not representative of the environment that the NPs are exposed to in vivo, it is important to understand whether insulin loading is affected by the presence of ions in the buffer. We therefore investigated the effect of buffer wash on insulin loading and NP size by preparing Ins-NPs and washing them either with DI water or with PBS buffer (pH 7.4 with $155 \mathrm{mM} \mathrm{NaCl}$ ) (Figure 1D). Ins-NPs washed only with DI water showed insulin loading of $8.95 \%$ (Figure 1E). By contrast, washing the NPs with PBS decreased the insulin loading by more than an order of magnitude to $0.36 \%$ (Figure 1E). The NP diameter also decreased slightly after PBS wash (from $65.2 \mathrm{~nm}$ to 62.8 $\mathrm{nm})$, but the change was not significant $(n=3, p=0.074)$ (Figure 1F). To understand 
whether the $\mathrm{pH}$ of the buffer had a stronger role in removing the weakly-bound insulin or the presence of $155 \mathrm{mM} \mathrm{NaCl}$ in the buffer, we washed the NPs with an unbuffered $155 \mathrm{mM}$ $\mathrm{NaCl}$ solution and the NPs showed a loading of $2.4 \%$ while washing the NPs with a pH 7.4 buffer without the $155 \mathrm{mM} \mathrm{NaCl}$ resulted in a loading of $0.85 \%$ (Supporting Information. Measurement of insulin loading in buffered and unbuffered solutions with varying $\mathrm{NaCl}$ concentration (Figure S3)).

The 25-fold decrease in insulin loading with minimal change in NP diameter upon buffer wash suggests that insulin in the case of NPs washed with DI water is primarily associated with the corona of the NPs via electrostatic and hydrophobic interactions where entropic factors could also be operative ${ }^{36,50}$. During a PBS wash, the NPs are exposed to $\mathrm{pH} 7.4$ (at which insulin is more soluble than unbuffered water), thereby facilitating release of insulin. Although $\mathrm{NaCl}$ does not play as large a role as $\mathrm{pH}$, it potentially facilitates removal of insulin by shielding electrostatic interactions. The results also suggest that some regions of insulin bind via its local positively and negatively charged patches to the corona of the NPs through electrostatic interactions, as seen in other negatively charged proteins like alactalbumin $^{51}$. Here, the partial negative charges on the polymer likely facilitate the interactions. Similar removal of loosely bound insulin from silica and chromium surfaces on rinsing with buffer has also been observed ${ }^{52-53}$. These results illustrate that washing of NPs using an appropriate solution is extremely important because it can dramatically alter the measured insulin loading. In the case of a DI water wash, the insulin loading of NPs is high, but it does not account for the effect of exposure to $\mathrm{pH}$ and salts. On the contrary, PBS wash gives a low loading of insulin but is more representative of in vivo environments. Therefore, we used the more conservative PBS wash in the rest of the study to assess the effect of various factors on insulin loading in NPs.

\subsection{Mechanism of NP formation}

The above results raise the question whether insulin simply adsorbs to the NP surface and plays little role in the process of nanoprecipitation. Such adsorption of small molecules on the surface of PLGA-PEG nanoparticles due to hydrophobic interactions has been reported recently ${ }^{54-55}$. If this hypothesis is true, we can expect the insulin loading to be similar regardless of whether insulin is added to the precursors during nanoprecipitation, or if it is added to the NPs after nanoprecipitation.

We therefore investigated the effect of the stage at which insulin is added during NP formation; specifically, we compared insulin loading of NPs formed by adding insulin along with PLGA-PEG before nanoprecipitation (Figure 2A (top), as described in section 2.2.1), with NPs formed by adding insulin to empty PLGA-PEG NPs after they were already formed by nanoprecipitation (Figure 2A (bottom)). Interestingly, similar insulin loading was obtained regardless of whether insulin was added before or after nanoprecipitation (Figure 2B). However, smaller NPs $(62.8 \mathrm{~nm})$ were produced when insulin was mixed with PLGAPEG before nanoprecipitation (Figure 2C). By contrast, larger NPs $(80.5 \mathrm{~nm}$ ) were formed when insulin was added to already-formed PLGA-PEG NPs; furthermore, their size was similar to that of empty PLGA-PEG NPs $(84 \mathrm{~nm})$. On studying the zeta potential of these 
NPs, we found that the zeta potential of Empty PLGA-PEG NPs and Ins-NPs was not significantly different (Supporting Information. Zeta potential measurement (Figure S4)).

The results only partially support the hypothesis that NP formation is independent of insulin - on one hand, we obtain similar insulin loading by simply adding insulin to already formed PLGA-PEG NPs, and on the other hand, the NPs are clearly smaller in size than PLGA-PEG NPs when insulin is added to DMSO along with PLGA-PEG before nanoprecipitation. This effect offers a potentially useful method to make smaller NPs; however, it also raises the question as to whether a similar reduction in NP size occurs if insulin is included in the aqueous phase instead of DMSO during nanoprecipitation (Figure 2D). We find that insulin loading is similar regardless of whether insulin was dissolved in water or in DMSO (Figure 2E), or added later to pre-formed NPs. However, dissolving insulin in the organic phase along with PLGA-PEG produced smaller sized NPs $(62.8 \mathrm{~nm})$ as compared to the NPs that were formed by including insulin in water $(84.9 \mathrm{~nm}$ ) (Figure 2F). Interestingly, the size of NPs formed by nanoprecipitation with insulin in the aqueous phase is similar to that of empty PLGA-PEG NPs without insulin, and to that of Ins-NPs formed by adding insulin to already formed PLGA-PEG NPs. The results show that insulin affects NP size only when it is dissolved in the organic phase (DMSO) along with PLGA-PEG, and in all other cases it does not change NP size compared to PLGA-PEG NPs without insulin.

The fact that nearly all of insulin is washed off by PBS, and that the insulin loading of NPs formed with insulin dissolved in water during nanoprecipitation is indistinguishable from insulin loading of NPs formed by adding insulin to PLGA-PEG NPs, indicates that insulin is loosely associated with the NP corona rather than encapsulated within the hydrophobic core. The fact that NPs can be loaded with insulin by simply adding insulin to NPs implies that NPs can be pre-formed to desired characteristics and subsequently loaded with insulin, thereby decoupling NP formation from insulin loading. It suggests that when insulin is included in the aqueous phase during nanoprecipitation or added after nanoprecipitation, its role in NP formation is passive - it simply adsorbs to the corona of the NPs and does not otherwise alter the NPs. However, when insulin is dissolved in the organic phase along with PLGA-PEG, it does influence the NP size, which presents a way to prepare smaller NPs compared to the corresponding PLGA-PEG NPs without insulin. This reduction in size of NPs when insulin is present during nanoprecipitation may be attributed to the hydrophilic nature of insulin that could act as a surfactant, potentially by associating with PLGA-PEG to stabilize smaller NPs and preventing their aggregation. These insights allow us to optimize the NP size and insulin loading, as illustrated below.

\subsection{Enhancing insulin loading by zinc chelation}

To improve insulin loading while retaining a small NP size, we took inspiration from previous studies that have shown an improvement in insulin adsorption on flat surfaces and particle systems (including PLGA-PEG micro and nanoparticles) by incorporating chelating ions like zinc into their systems ${ }^{33}, 36,52-53,56$. This increase in adsorption is attributed to the formation of stable insulin-zinc (Ins-Zn) hexamers ${ }^{56-57}$. In fact, it has been shown in previous studies that the presence of zinc enhances insulin stability and is widely used in the formulation of long acting insulin ${ }^{58}$. Moreover, studies have also reported the non-cytotoxic 
efficacy of PLGA-PEG microparticles with zinc and insulin as payload ${ }^{36,59}$. Zinc exposure and consumption is also known to be safe as shown in a study done with 24 volunteers with 2-35.5 years of exposure to $₫ 30 \mathrm{mg}$ zinc/ $\mathrm{m}^{3}$ as metallic zinc dust, zinc sulfide dust, zinc oxide reported no nausea or vomiting ${ }^{60}$.

Ins-Zn hexamer formation first involves the formation of insulin dimers, followed by the assembly of two dimers with two zinc ions to give a tetramer $\left(\mathrm{Zn}^{2+}\right)_{2}(\mathrm{In})_{4}$, which then combines with another dimeric unit to give an insulin hexamer $\left(\mathrm{Zn}^{2+}\right)_{2}(\mathrm{In})_{6}$ (Figure 3A $($ top $))^{61}$. The tendency for protein adsorption is generally expected to increase with molecular weight and hydrophobicity of the protein ${ }^{61}$. Moreover, the strength of a polyvalent interaction (like Ins-Zn hexamer with the NP surface) can be much stronger than that which can be achieved by a fewer number of interactions (like Ins monomer with NP corona) ${ }^{62-64}$.. Given the larger size of the Ins-Zn hexamer and its lower solubility in water ${ }^{65}$, we expect that incorporation of zinc ions in the Ins-NP system will improve insulin loading. Previous studies have also suggested that the presence of zinc ions ensures the integrity of the functional secondary structure of insulin, thereby maintaining its bioactivity ${ }^{36,56}$. Similar results have also been shown for other proteins like human growth hormone and human nerve growth factor ${ }^{36}$.

We therefore investigated the effect of including zinc ions in the organic phase along with insulin at different molar ratios (1:3, 1:6, 1:9 of Ins:Zn) to form Ins-Zn-NPs, where the stoichiometry for a hexamer is 1:3 (Figure 3A). TEM EDX analysis confirmed the presence of zinc ions in the resulting NPs (Figure 3B), where the zinc signal was co-localized with that from the PLGA-PEG NPs. We observed a monotonic increase in insulin loading as the amount of zinc ions was increased in the NPs while maintaining the same insulin and PLGA-PEG concentrations (Figure 3C). At an Ins:Zn molar ratio of 1:9, the NPs showed more than 10-fold higher insulin loading (4.07\%) as compared to Ins-NPs without zinc $(0.36 \%)$. On measuring the hydrodynamic diameter of Ins-Zn-NPs, we observed that the size of Ins-Zn-NPs (127.6 nm) was significantly greater than the size of Ins-NPs $(62.8 \mathrm{~nm})$ and also the size of empty PLGA-PEG NPs ( $84 \mathrm{~nm}$ ) (Figure 3D). The zeta potential of Ins-Zn NPs $(-22.03 \mathrm{mV})$ was also significantly lower as compared to the zeta potential of empty NPs (-25.44 mV) and Ins-NPs (-27.9mV) (Supporting Information. Zeta potential measurement (Figure S4)), showing that the zinc ions decreased the surface charge of the NPs, presumably by binding to negative charges on insulin. However, the size of these NPs was independent of the amount of zinc ions added to the system. This increase in size of the NPs is expected since the hexameric form of insulin is less soluble in the aqueous phase as compared to its monomeric form, which could promote NP aggregation and lead to larger NPs. Moreover, the presence of zinc ions in the NPs did not affect the conformational integrity of insulin, as confirmed by circular dichroism spectroscopy ${ }^{36}$ (Supporting Information. Measurement of preserved secondary structure of insulin after encapsulation and release from NPs using circular dichroism (Figure S5d)).

\subsection{Optimizing the size of Ins-Zn-NPs}

Based upon our earlier understanding of the Ins-NPs (Section 3.2), we hypothesized that it would be possible to generate sub-100 nm NPs with high insulin loading by adding zinc or 
Ins-Zn to already-formed PLGA-PEG NPs. NP characteristics of Ins-Zn-NPs, synthesized by adding Ins-Zn hexamers mixed in DMSO along with PLGA-PEG and added before nanoprecipitation (Figure 4A), were compared with Ins-Zn-NPs formed by adding Ins-Zn hexamers to pre-formed PLGA-PEG NPs (Figure 4B) and Ins-Zn-NPs formed by adding zinc ions to preformed Ins-NPs (Figure 4C).

Ins-Zn-NPs formed by adding zinc ions to already synthesized Ins-NPs (Figure 4C), showed a reduction in NP size from $127.6 \mathrm{~nm}$ to $57.9 \mathrm{~nm}$ (similar to size of Ins-NPs), but the insulin loading decreased from $4.07 \%$ to $1.55 \%$ (Figure $4 \mathrm{~A}$ and $4 \mathrm{C}$ ). When Ins-Zn hexamers were added after assembling empty PLGA-PEG NPs by nanoprecipitation, the resulting Ins-ZnNPs were $78.7 \mathrm{~nm}$ in diameter (similar to the size of empty PLGA-PEG NPs) while maintaining more than 10-fold higher insulin loading (3.82 \%) compared to Ins-NPs. Here, we again observe that addition of Ins-Zn hexamers (or zinc ions) to pre-formed empty PLGA-PEG NPs (or pre-formed Ins-NPs) does not change the hydrodynamic diameter of those NPs. However, the size of the Ins-Zn-NPs $(\sim 120 \mathrm{~nm})$ formed by including Ins-Zn in the organic phase (DMSO) is much larger than that of PLGA-PEG NPs $(\sim 80 \mathrm{~nm})$, analogous to, but opposite to the effect of including insulin in DMSO during formation of Ins-NPs. These results point to a general principle, where addition of insulin to pre-formed NPs enables insulin loading without affecting NP size, but including insulin in the same solution as the polymeric precursor can influence NP size.

\subsection{Effect of $\mathrm{pH}$ on formation of Ins-Zn-NPs}

Insulin exhibits $\mathrm{pH}$-dependent behavior with an isoelectric point of $5.6^{66}$, which imparts insulin a net negative charge at $\mathrm{pH}$ greater than $\mathrm{pH} 5.6$ and a net positive charge at $\mathrm{pH}$ less than 5.6. Since our findings suggest that the synthesis of Ins-NPs by nanoprecipitation could be affected partially by electrostatic interactions, we expect to be able to control the insulin loading by changing the net charge on insulin by altering the $\mathrm{pH}$ of the system.

To better understand the role of $\mathrm{pH}$, we synthesized Ins-Zn-NPs in three different buffers: acetate buffer (20 mM, pH 4.65), MES buffer (20 mM, pH 6.1) and phosphate buffer (20 $\mathrm{mM}, \mathrm{pH}$ 6.5) (Figure 5A). In the case of Ins-Zn-NPs, insulin loading increases with increasing $\mathrm{pH}$ (Figure 5B), which can be attributed to the larger net negative charge on insulin at $\mathrm{pH}$ greater than 5.6, which enables stronger interactions with insulin and zinc ions ${ }^{36}$. Ins-Zn-NPs prepared at $\mathrm{pH} 6.5$ showed higher insulin loading (4.7\%) than the InsZn-NPs prepared in DI water (4.07\%). Therefore, by optimizing the $\mathrm{pH}$ of the system, insulin loading can further be improved in PLGA-PEG NPs. A similar trend was observed in the case of Ins-NP without zinc, where insulin loading slightly increased with increasing $\mathrm{pH}$ (Figure 5B). It is noteworthy that at $\mathrm{pH} 4.65$, zinc ions did not have any effect on insulin loading in PLGA-PEG NPs, and the insulin loading both with and without zinc was similar $(\sim 0.2 \%)$. This observation can be attributed to the net positive charge on insulin below $\mathrm{pH}$ 5.6 that leads to a reduction in the interactions between insulin and zinc and makes the Ins$\mathrm{Zn}$ hexamers unstable below pH 5.6 $6^{36}$. By contrast to insulin loading, $\mathrm{pH}$ does not have a significant effect on the size of Ins-Zn-NPs (Figure 5C). 


\subsection{Optimization of NPs for small size and high insulin loading}

The key factors that affect NP characteristics and how they can be altered to control two important NP characteristics - insulin loading and NP size - are summarized in Figure 6A and 6B. It is evident from the representation in these figures that Ins-NPs are smaller in size but show poor insulin loading, while the inclusion of zinc in these NPs during nanoprecipitation helps to increase the insulin loading but results in an increase in the size of the NPs. We also see that addition of insulin at different stages and mixing insulin in different phases affects NP size but does not change the insulin loading. This behavior was exploited in optimizing the insulin loading and size of Ins-Zn-NPs. On addition of zinc ions to pre-formed Ins-NPs, insulin loading increased 4-fold (1.55\% from $0.36 \%$ ) without resulting in an increase in the size of Ins-NP $(57.9 \mathrm{~nm})$. Furthermore, the strategic inclusion of insulin-zinc hexamers to pre-formed empty PLGA-PEG NPs results in a greater than 10fold high insulin loading (3.82\%) and small NP size $(78.7 \mathrm{~nm})$, thus making them promising NPs for oral delivery of insulin using a transcytosis pathway like the FcRn pathway.

Figure 6C shows the rate at which insulin is released from the Ins-Zn-NPs (where Ins-Zn is added later; Insulin loading $3.82 \%$, NP size $78.7 \mathrm{~nm}$ ). The half-life of insulin in these NPs is around $60 \mathrm{~min}$. The release curve of Ins-Zn-NPs is similar to the insulin loaded PLA-PEG NPs release curve which was reported in an earlier study where the insulin loading in PLAPEG NPs was about $0.5 \%$.

\section{Conclusions}

Realizing the full potential of polymeric NP based protein therapeutics will require more efficient loading of proteins in these NPs. To understand the factors that control protein loading in NPs formed by nanoprecipitation, we systematically investigated the effect of the washing step, choice of the solvent in which insulin is added, and the stage at which insulin is added to the system. First, by controlling these parameters, we have shown that the type of wash can dramatically influence the measured insulin loading in Ins-NPs, where buffer wash removes majority of the insulin compared to water wash. Second, we also demonstrate that smaller Ins-NPs can be formed by dissolving insulin along with the PLGA-PEG molecules in organic phase (DMSO) before nanoprecipitation. In the case of Ins-Zn-NP system, more than 10-fold increase in insulin loading compared to Ins-NPs was obtained by using Ins-Zn hexamers. We also applied the insights obtained from the Ins-NP system to Ins-Zn-NP system to achieve small NP diameter while maintaining high insulin loading. In particular, we find that insulin and/or zinc may be added to pre-formed NPs to load insulin without affecting the NP size. Third, in addition, insulin loading in Ins-Zn-NP system could be optimized by tuning the $\mathrm{pH}$ of the system. This study illustrates how simple modifications of the nanoprecipitation process can provide insights that enable realization of NPs with desired properties. This approach can potentially extend to other protein-encapsulating NP systems to understand the mechanisms of protein loading and to devise processes to enhance protein loading in NPs formed by nanoprecipitation.

\section{Supplementary Material}

Refer to Web version on PubMed Central for supplementary material. 


\section{Acknowledgments}

The authors thank Professor Robert Langer (MIT) for his support and access to experimental equipment, Dr. Archana Swami (Harvard Medical School), Dr. Suman Bose (MIT), and Abel Cortinas for helpful discussions, and Dr. Yun (MIT) for help with TEM EDX. TEM imaging was performed in part at the Koch Institute imagining facility at MIT and at the Center for Materials Science and Engineering (CMSE) at MIT, a MRSEC Shared Experimental Facilities supported by the National Science Foundation under award number DMR-0819762. N.B acknowledges a postdoctoral fellowship from the Canadian Institutes of Health Research. This research was supported by NIH Grant R01 EB015419 (O.C.F. and R.K.).

\section{References}

1. Frokjaer S, Otzen DE. Protein Drug Stability: A Formulation Challenge. Nat Rev Drug Discovery. 2005; 4(4):298-306. [PubMed: 15803194]

2. Baumann A. Early Development of Therapeutic Biologics-Pharmacokinetics. Curr Drug Metab. 2006; 7(1):15-21. [PubMed: 16454690]

3. Singh JA, Christensen R, Wells GA, Suarez-Almazor ME, Buchbinder R, Lopez-Olivo MA, Ghogomu ET, Tugwell P. Biologics for Rheumatoid Arthritis: An Overview of Cochrane Reviews. Rev Paul Med. 2010; 128(5):309-310.

4. Quesada JR, Talpaz M, Rios A, Kurzrock R, Gutterman J. Clinical Toxicity of Interferons in Cancer Patients: A Review. J Clin Oncol. 1986; 4(2):234-243. [PubMed: 2418169]

5. Rossini AA, Mordes JP, Like AA. Immunology of Insulin-Dependent Diabetes Mellitus. Annu Rev Immunol. 1985; 3(1):289-320. [PubMed: 3904771]

6. Pridgen EM, Alexis F, Kuo TT, Levy-Nissenbaum E, Karnik R, Blumberg RS, Langer R, Farokhzad OC. Transepithelial Transport of Fc-Targeted Nanoparticles by the Neonatal Fc Receptor for Oral Delivery. Sci Transl Med. 2013; 5(213)

7. Sonia T, Sharma CP. An Overview of Natural Polymers for Oral Insulin Delivery. Drug discovery today. 2012; 17(13):784-792. [PubMed: 22521664]

8. Mitragotri S, Burke PA, Langer R. Overcoming the Challenges in Administering Biopharmaceuticals: Formulation and Delivery Strategies. Nat Rev Drug Discovery. 2014; 13(9): 655-672. [PubMed: 25103255]

9. Ibraheem D, Elaissari A, Fessi H. Administration Strategies for Proteins and Peptides. Int J Pharm. 2014; 477(1):578-589. [PubMed: 25445533]

10. Brigger I, Dubernet C, Couvreur P. Nanoparticles in Cancer Therapy and Diagnosis. Adv Drug Delivery Rev. 2002; 54(5):631-651.

11. Bertrand N, Wu J, Xu X, Kamaly N, Farokhzad OC. Cancer Nanotechnology: The Impact of Passive and Active Targeting in the Era of Modern Cancer Biology. Adv Drug Delivery Rev. 2014; 66:2-25.

12. Khalil NM, Carraro E, Cótica LF, Mainardes RM. Potential of Polymeric Nanoparticles in AIDS Treatment and Prevention. Expert Opin Drug Delivery. 2011; 8(1):95-112.

13. Santos-Magalhães NS, Mosqueira VCF. Nanotechnology Applied to the Treatment of Malaria. Adv Drug Delivery Rev. 2010; 62(4):560-575.

14. Kattan J, Droz JP, Couvreur P, Marino JP, Boutan-Laroze A, Rougier P, Brault P, Vranckx H, Grognet JM, Morge X. Phase I Clinical Trial and Pharmacokinetic Evaluation of Doxorubicin Carried by Polyisohexylcyanoacrylate Nanoparticles. Invest New Drugs. 1992; 10(3):191-199. [PubMed: 1428729]

15. Lee KS, Chung HC, Im SA, Park YH, Kim CS, Kim SB, Rha SY, Lee MY, Ro J. Multicenter Phase II Trial of Genexol-PM, a Cremophor-Free, Polymeric Micelle Formulation of Paclitaxel, in Patients with Metastatic Breast Cancer. Breast Cancer Res Treat. 2008; 108(2):241-250. [PubMed: 17476588]

16. Hrkach J, Von Hoff D, Ali MM, Andrianova E, Auer J, Campbell T, De Witt D, Figa M, Figueiredo M, Horhota A. Preclinical Development and Clinical Translation of a PSMA-Targeted Docetaxel Nanoparticle with a Differentiated Pharmacological Profile. Sci Transl Med. 2012; 4(128) 
17. Kamaly N, Xiao Z, Valencia PM, Radovic-Moreno AF, Farokhzad OC. Targeted Polymeric Therapeutic Nanoparticles: Design, Development and Clinical Translation. Chem Soc Rev. 2012; 41(7):2971-3010. [PubMed: 22388185]

18. Shi J, Xiao Z, Kamaly N, Farokhzad OC. Self-Assembled Targeted Nanoparticles: Evolution of Technologies and Bench to Bedside Translation. Acc Chem Res. 2011; 44(10):1123-1134. [PubMed: 21692448]

19. Kumari A, Yadav SK, Yadav SC. Biodegradable Polymeric Nanoparticles Based Drug Delivery Systems. Colloids Surf, B. 2010; 75(1):1-18.

20. Chen MC, Sonaje K, Chen KJ, Sung HW. A Review of the Prospects for Polymeric Nanoparticle Platforms in Oral Insulin Delivery. Biomaterials. 2011; 32(36):9826-9838. [PubMed: 21925726]

21. Martin-Banderas L, Duran-Lobato M, Munoz-Rubio I, Alvarez-Fuentes J, Fernandez-Arevalo M, Holgado MA. Functional PLGA NPs for Oral Drug Delivery: Recent Strategies and Developments. Mini-Rev Med Chem. 2013; 13(1):58-69. [PubMed: 22974367]

22. Danhier F, Ansorena E, Silva JM, Coco R, Le Breton A, Préat V. PLGA-Based Nanoparticles: An Overview of Biomedical Applications. J Controlled Release. 2012; 161(2):505-522.

23. Elsabahy M, Wooley KL. Design of Polymeric Nanoparticles for Biomedical Delivery Applications. Chem Soc Rev. 2012; 41(7):2545-2561. [PubMed: 22334259]

24. Pridgen EM, Alexis F, Farokhzad OC. Polymeric Nanoparticle Drug Delivery Technologies for Oral Delivery Applications. Expert Opin Drug Delivery. 2015; 12(9):1459-1473.

25. Fuhrmann G, Grotzky A, Luki` R, Matoori S, Luciani P, Yu H, Zhang B, Walde P, Schlüter AD, Gauthier MA. Sustained Gastrointestinal Activity of Dendronized Polymer-Enzyme Conjugates. Nat Chem. 2013; 5(7):582-589. [PubMed: 23787748]

26. Raghuvanshi RS, Katare YK, Lalwani K, Ali MM, Singh O, Panda AK. Improved Immune Response from Biodegradable Polymer Particles Entrapping Tetanus Toxoid by Use of Different Immunization Protocol and Adjuvants. Int J Pharm. 2002; 245(1):109-121. [PubMed: 12270248]

27. Satheesh Kumar P, Ramakrishna S, Ram Saini T, Diwan P. Influence of Microencapsulation Method and Peptide Loading on Formulation of Poly (lactide-co-glycolide) Insulin Nanoparticles. Pharmazie. 2006; 61(7):613-617. [PubMed: 16889069]

28. Park J, Wrzesinski SH, Stern E, Look M, Criscione J, Ragheb R, Jay SM, Demento SL, Agawu A, Limon PL. Combination Delivery of TGF- $\beta$ Inhibitor and IL-2 by Nanoscale Liposomal Polymeric Gels Enhances Tumour Immunotherapy. Nat Mater. 2012; 11(10):895-905. [PubMed: 22797827]

29. des Rieux A, Fievez V, Garinot M, Schneider YJ, Préat V. Nanoparticles as Potential Oral Delivery Systems of Proteins and Vaccines: A Mechanistic Approach. J Controlled Release. 2006; 116(1): $1-27$.

30. Makadia HK, Siegel SJ. Poly (lactic-co-glycolic acid) (PLGA) as Biodegradable Controlled Drug Delivery Carrier. Polymers (Basel, Switz). 2011; 3(3):1377-1397.

31. Pandey, SK., Haldar, C., Patel, DK., Maiti, P. Multifaceted Dev App Biopoly Bio, Biomed, Nanotech. Springer; 2013. Biodegradable Polymers for Potential Delivery Systems for Therapeutics; p. 169-202.

32. Hans M, Lowman A. Biodegradable Nanoparticles for Drug Delivery and Targeting. Curr Opin Solid State Mater Sci. 2002; 6(4):319-327.

33. Jain S, Rathi VV, Jain AK, Das M, Godugu C. Folate-Decorated PLGA Nanoparticles as a Rationally Designed Vehicle for the Oral Delivery of Insulin. Nanomedicine. 2012; 7(9):13111337. [PubMed: 22583576]

34. Mittal G, Sahana D, Bhardwaj V, Kumar MR. Estradiol Loaded PLGA Nanoparticles for Oral Administration: Effect of Polymer Molecular Weight and Copolymer Composition on Release Behavior In Vitro and In Vivo. J Controlled Release. 2007; 119(1):77-85.

35. Hirsch IB. Type 1 Diabetes Mellitus and the Use of Flexible Insulin Regimens. Am Fam Physician. 1999; 60(8):2343-2352. [PubMed: 10593324]

36. Manoharan C, Singh J. Insulin Loaded PLGA Microspheres: Effect of Zinc Salts on Encapsulation, Release, and Stability. J Pharm Sci. 2009; 98(2):529-542. [PubMed: 18548615]

37. Malathi S, Nandhakumar P, Pandiyan V, Webster TJ, Balasubramanian S. Novel PLGA-Based Nanoparticles for the Oral Delivery of Insulin. Int J Nanomed. 2015; 10:2207-2218. 
38. Sah E, Sah H. Recent Trends in Preparation of Poly (lactide-co-glycolide) Nanoparticles by Mixing Polymeric Organic solution with Antisolvent. J Nanomater. 2015; 16(1):61.

39. Cheng J, Teply BA, Sherifi I, Sung J, Luther G, Gu FX, Levy-Nissenbaum E, Radovic-Moreno AF, Langer R, Farokhzad OC. Formulation of Functionalized PLGA-PEG Nanoparticles for In Vivo Targeted Drug Delivery. Biomaterials. 2007; 28(5):869-876. [PubMed: 17055572]

40. Ahlin Grabnar P, Kristl J. The Manufacturing Techniques of Drug-Loaded Polymeric Nanoparticles from Preformed Polymers. J Microencapsulation. 2011; 28(4):323-335. [PubMed: 21545323]

41. Mora-Huertas C, Fessi H, Elaissari A. Polymer-Based Nanocapsules for Drug Delivery. Int J Pharm (Amsterdam, Neth). 2010; 385(1):113-142.

42. Avgoustakis K. Pegylated Poly (lactide) and Poly (lactide-co-glycolide) Nanoparticles: Preparation, Properties and Possible Applications in Drug Delivery. Curr Drug Delivery. 2004; 1(4):321-333.

43. Brayton CF. Dimethyl Sulfoxide (DMSO): a Review. Cornell Vet. 1986; 76(1):61-90. [PubMed: 3510103]

44. Lopes MA, Abrahim-Vieira B, Oliveira C, Fonte P, Souza AM, Lira T, Sequeira JA, Rodrigues CR, Cabral LM, Sarmento B. Probing Insulin Bioactivity in Oral Nanoparticles Produced by Ultrasonication-Assisted Emulsification/Internal Gelation. Int J Nanomed. 2015; 10:5865-5880.

45. Tiyaboonchai W, Woiszwillo J, Sims RC, Middaugh CR. Insulin Containing PolyethylenimineDextran Sulfate Nanoparticles. Int J Pharm (Amsterdam, Neth). 2003; 255(1):139-151.

46. Chou DHC, Webber MJ, Tang BC, Lin AB, Thapa LS, Deng D, Truong JV, Cortinas AB, Langer R, Anderson DG. Glucose-Responsive Insulin Activity by Covalent Modification with Aliphatic Phenylboronic Acid Conjugates. Proc Natl Acad Sci U S A. 2015; 112(8):2401-2406. [PubMed: 25675515]

47. Naha PC, Kanchan V, Manna P, Panda AK. Improved Bioavailability of Orally Delivered Insulin Using Eudragit-L30D Coated PLGA Microparticles. J Microencapsulation. 2008; 25(4):248-256. [PubMed: 18465311]

48. Tomar L, Tyagi C, Kumar M, Kumar P, Singh H, Choonara YE, Pillay V. In Vivo Evaluation of a Conjugated Poly (lactide-ethylene glycol) Nanoparticle Depot Formulation for Prolonged Insulin Delivery in the Diabetic Rabbit Model. Int J Nanomedicine. 2013; 8:505-520. [PubMed: 23429428]

49. Cózar-Bernal M, Holgado M, Arias J, Muñoz-Rubio I, Martín-Banderas L, Álvarez-Fuentes J, Fernández-Arévalo M. Insulin-Loaded PLGA Microparticles: Flow Focusing Versus Double Emulsion/Solvent Evaporation. J Microencapsulation. 2011; 28(5):430-441. [PubMed: 21736527]

50. Roach P, Farrar D, Perry CC. Interpretation of Protein Adsorption: Surface-Induced Conformational Changes. J Am Chem Soc. 2005; 127(22):8168-8173. [PubMed: 15926845]

51. Pasche S, Voros J, Griesser HJ, Spencer ND, Textor M. Effects of Ionic Strength and Surface Charge on Protein Adsorption at PEGylated Surfaces. J Phys Chem B. 2005; 109(37):1754517552. [PubMed: 16853244]

52. Arnebrant T, Nylander T. Adsorption of Insulin on Metal Surfaces in Relation to Association Behavior. J Colloid Interface Sci. 1988; 122(2):557-566.

53. Nilsson P, Nylander T, Havelund S. Adsorption of Insulin on Solid Surfaces in Relation to the Surface Properties of the Monomeric and Oligomeric Forms. J Colloid Interface Sci. 1991; 144(1): $145-152$.

54. Brandl F, Bertrand N, Lima EM, Langer R. Nanoparticles with Photoinduced Precipitation for the Extraction of Pollutants from Water and Soil. Nat Commun. 2015; 6:7765. [PubMed: 26196119]

55. Monopoli MP, Åberg C, Salvati A, Dawson KA. Biomolecular Coronas Provide the Biological Identity of Nanosized Materials. Nat Nanotechnol. 2012; 7(12):779-786. [PubMed: 23212421]

56. Malathi S, Nandhakumar P, Pandiyan V, Webster TJ, Balasubramanian S. Novel PLGA-Based Nanoparticles for the Oral Delivery of Insulin. Int J Nanomed. 2015; 10:2207.

57. Marcker K. The Binding of the "Structural" Zinc Ions in Crystalline Insulin. Acta Chem Scand (1947-1973). 1960; 14:2071-2074.

58. Havelund S, Plum A, Ribel U, Jonassen I, Vølund A, Markussen J, Kurtzhals P. The Mechanism of Protraction of Insulin Detemir, A Long-Acting, Acylated Analog of Human Insulin. Pharm Res. 2004; 21(8):1498-1504. [PubMed: 15359587] 
59. Jain S, Rathi VV, Jain A, Das M, Godugu C. Folate-Decorated PLGA Nanoparticles as a Rationally Designed Vehicle for the Oral Delivery of Insulin. Nanomedicine. 2012; 7(9):13111337. [PubMed: 22583576]

60. Toxicological Profile for Zinc. US Department of Health and Human Services; 2005.

61. Dunn MF. Zinc-Ligand Interactions Modulate Assembly and Stability of the Insulin Hexamer-A Review. Biometals : an international journal on the role of metal ions in biology, biochemistry, and medicine. 2005; 18(4):295-303.

62. Mammen M, Choi SK, Whitesides GM. Polyvalent Interactions in Biological Systems: Implications for Design and Use of Multivalent Ligands and Inhibitors. Angew Chem, Int Ed. 1998; 37(20):2754-2794.

63. Arai T, Norde W. The Behavior of Some Model Proteins at Solid-Liquid Interfaces 1. Adsorption from Single Protein Solutions. Colloids Surf. 1990; 51:1-15.

64. Marcker K. Association of Zn-Free Insulin. Acta Chem Scand (1947-1973). 1960; 14(1)

65. Hallas-Møller K, Petersen K, Schlichtkrull J. Crystalline and Amorphous Insulin-Zinc Compounds with Prolonged Action. Science (Washington, DC, U S). 1952; 116(3015):394-398.

66. Wintersteiner O, Abramson HA. The Isoelectric Point of Insulin Electrical Properties of Adsorbed and Crystalline Insulin. J Biol Chem. 1933; 99(3):741-753. 
(A)
(B)

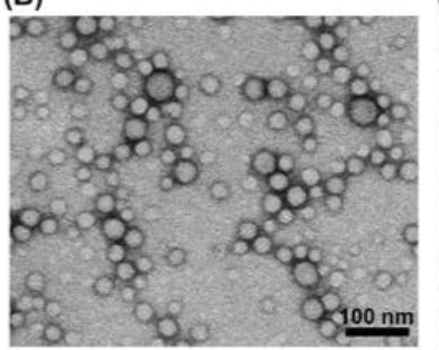

(F)
(C)

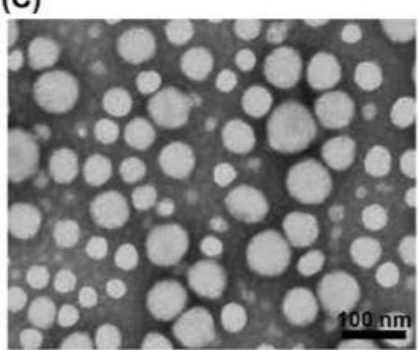

(E)

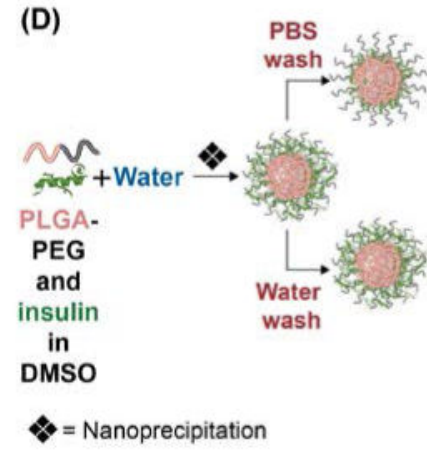

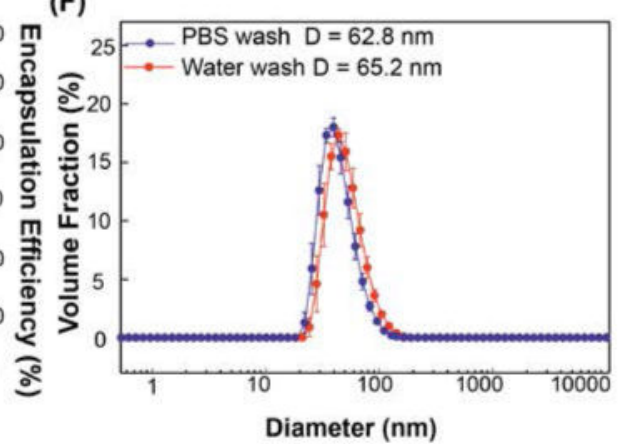

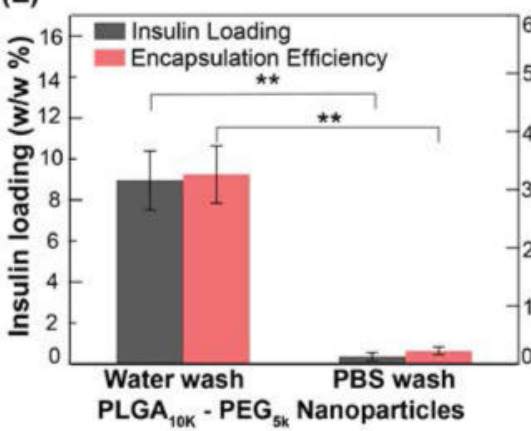

Figure 1. Insulin loaded PLGA-PEG nanoparticles (Ins-NP)

(A) Schematic of Ins-NP synthesis process by nanoprecipitation, where PLGA-PEG and insulin in dimethyl sulfoxide (DMSO) is added drop-wise to a stirred beaker containing water (or buffer). TEM images of (B) Ins-NPs and (C) PLGA-PEG NPs without insulin. Effect of buffer wash on Ins-NP characteristics. (D) Schematic of the synthesis of Ins-NPs where NPs were either washed with PBS buffer (pH 7.4) or with water only. (E) Insulin loading and encapsulation efficiency show 25 -fold higher insulin loading when NPs were washed with water only $(8.95 \% \pm 1.44)$ as compared to a PBS buffer wash $(0.36 \% \pm 0.19)(n$ $=3, p=0.008$ ). (F) NP size data show no significant difference in the size of the Ins-NPs when washed with water or buffer $(n=3, p=0.074)$. Error bars represent \pm s.d. 
(A)

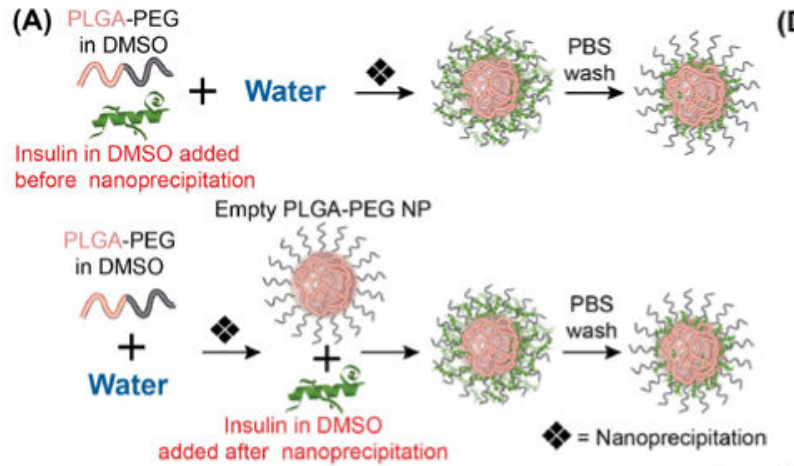

(B)

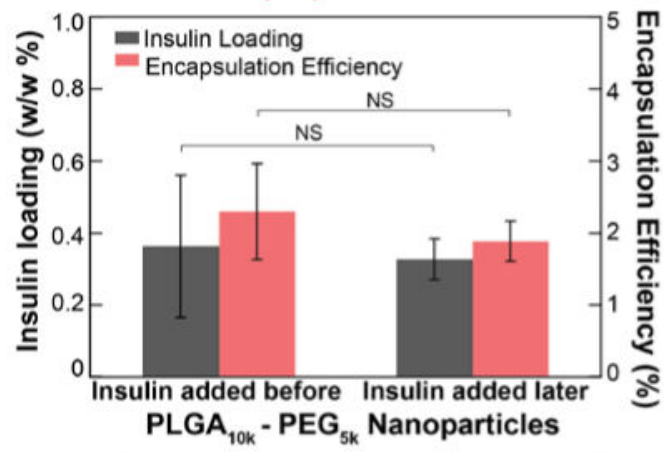

(C)

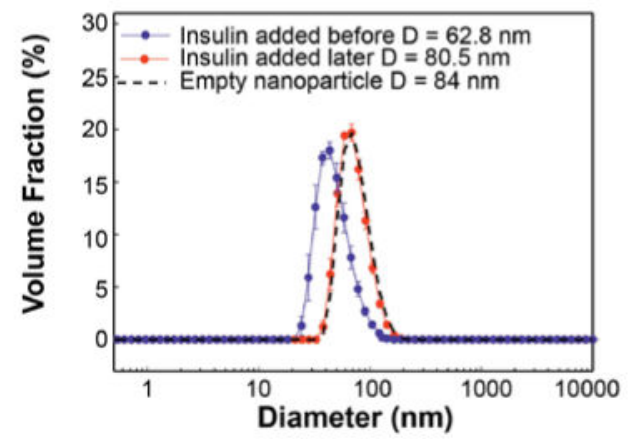

(D)

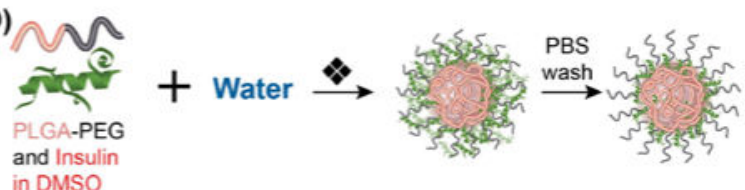

in DMSO

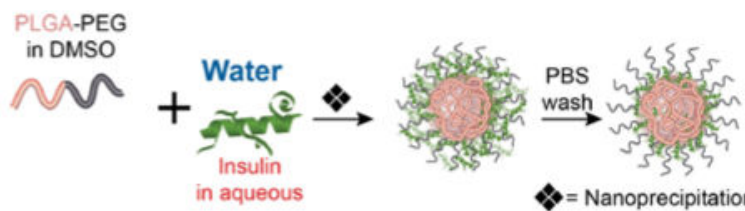

(E)

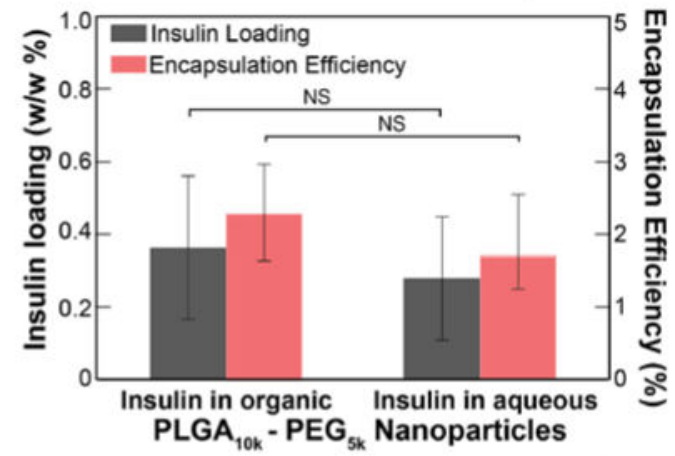

(F)

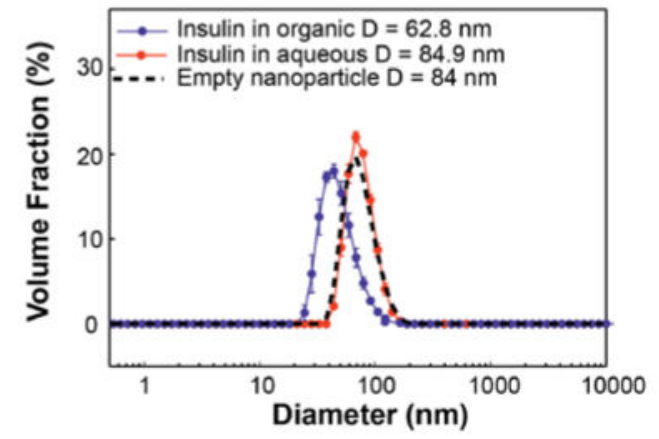

Figure 2. Effect of adding insulin at different stages of nanoprecipitation on Ins-NP characteristics

(A) Schematic showing the synthesis of Ins-NPs by including insulin mixed with PLGAPEG in DMSO before nanoprecipitation (top), and by adding insulin in DMSO to empty PLGA-PEG NPs after their formation by nanoprecipitation (bottom). (B) Insulin loading and encapsulation efficiency show no significant dependence on the stage of adding insulin before $(0.36 \%)$ or after $(0.32 \%)$ nanoprecipitation $(n=3, p=0.79)$, but (C) NP size shows a significant dependence $(n=3, p=0.005)$. Effect of mixing insulin in different phases on InsNP characteristics. (D) Schematic showing the synthesis of Ins-NPs by adding DMSO with insulin and PLGA-PEG to water (top), and by adding DMSO with PLGA-PEG to water with insulin dissolved in it (bottom). (E) Insulin loading (and encapsulation efficiency) shows no significant dependence on including insulin in DMSO $(0.36 \%)$ or in water $(0.28 \%)(n=3, p$ $=0.60)$, but $(\mathrm{F}) \mathrm{NP}$ size shows a significant dependence $(n=3, p=0.0006)$. Error bars represent \pm s.d. Note: The NPs with the label 'Insulin added before' in Figure 2B and the NPs with the label 'Insulin in organic' in Figure 2E are the same nanoparticles. 
(B)

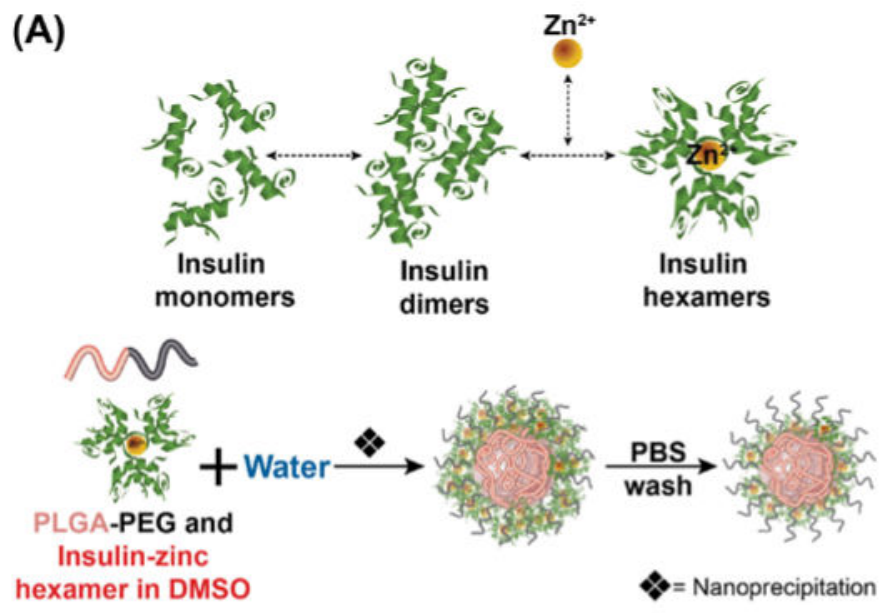

\section{(A)}

(C)

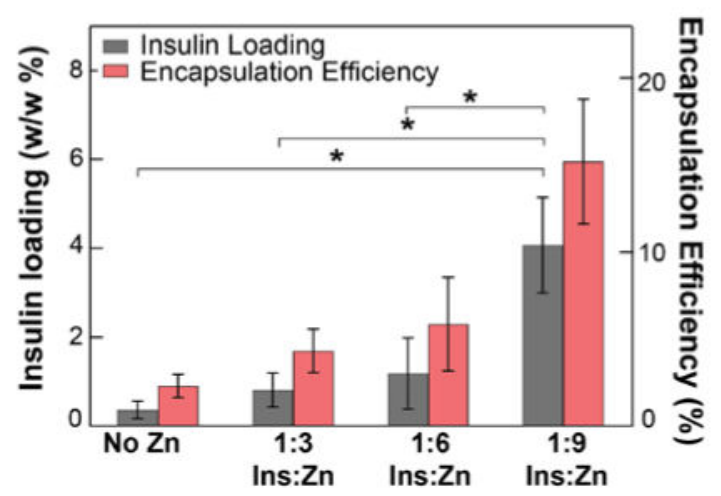

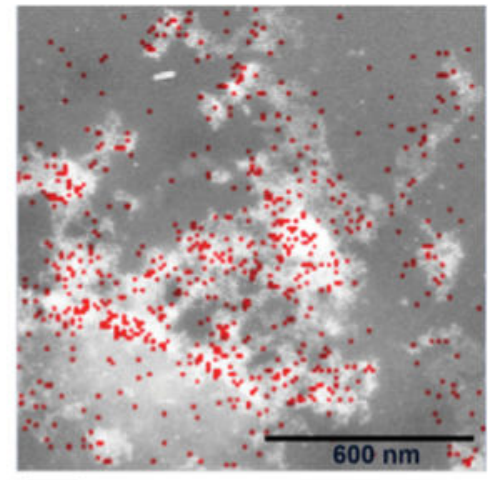

Insulin-Zn PLGA-PEG NPs

Zinc

(D)

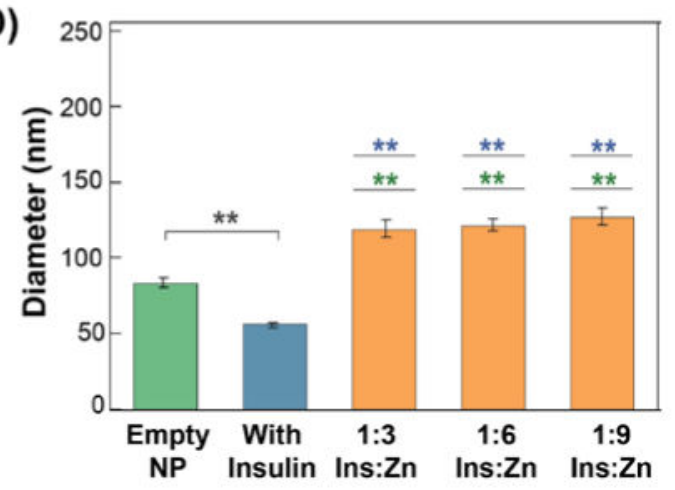

Figure 3. Effect of incorporating zinc ions in Ins-NPs

(A) Schematic showing the formation of Ins-Zn hexamers (top) and of Ins-Zn-NPs (bottom).

(B) Dark-field TEM/EDX image of unstained Ins-Zn-NPs. The lighter regions in the figure show the presence of NPs, and the red dots correspond to the presence of zinc. The presence of zinc in the same areas where Ins-Zn-NPs were detected confirms the presence of zinc in Ins-Zn-NPs. (C) Insulin loading and encapsulation efficiency show an increase in insulin loading with an increase in zinc ion concentration. Greater than 10-fold enhancement of insulin loading was obtained for insulin to zinc ratio of 1:9. $(n=3, p=0.02)$. (D) The average diameter of empty PLGA-PEG NPs, Ins-NPs, and Ins-Zn-NPs. In case of Ins-ZnNPs, the average diameter did not change significantly when different amount of zinc ions are added. The average diameter of Ins-Zn-NPs was larger than that of empty PLGA-PEG NPs $(n=3, p=0.0003)$ and Ins-Zn-NPs and Ins-NPs $(n=3, p=0.0001)$. Error bars represent \pm s.d. 


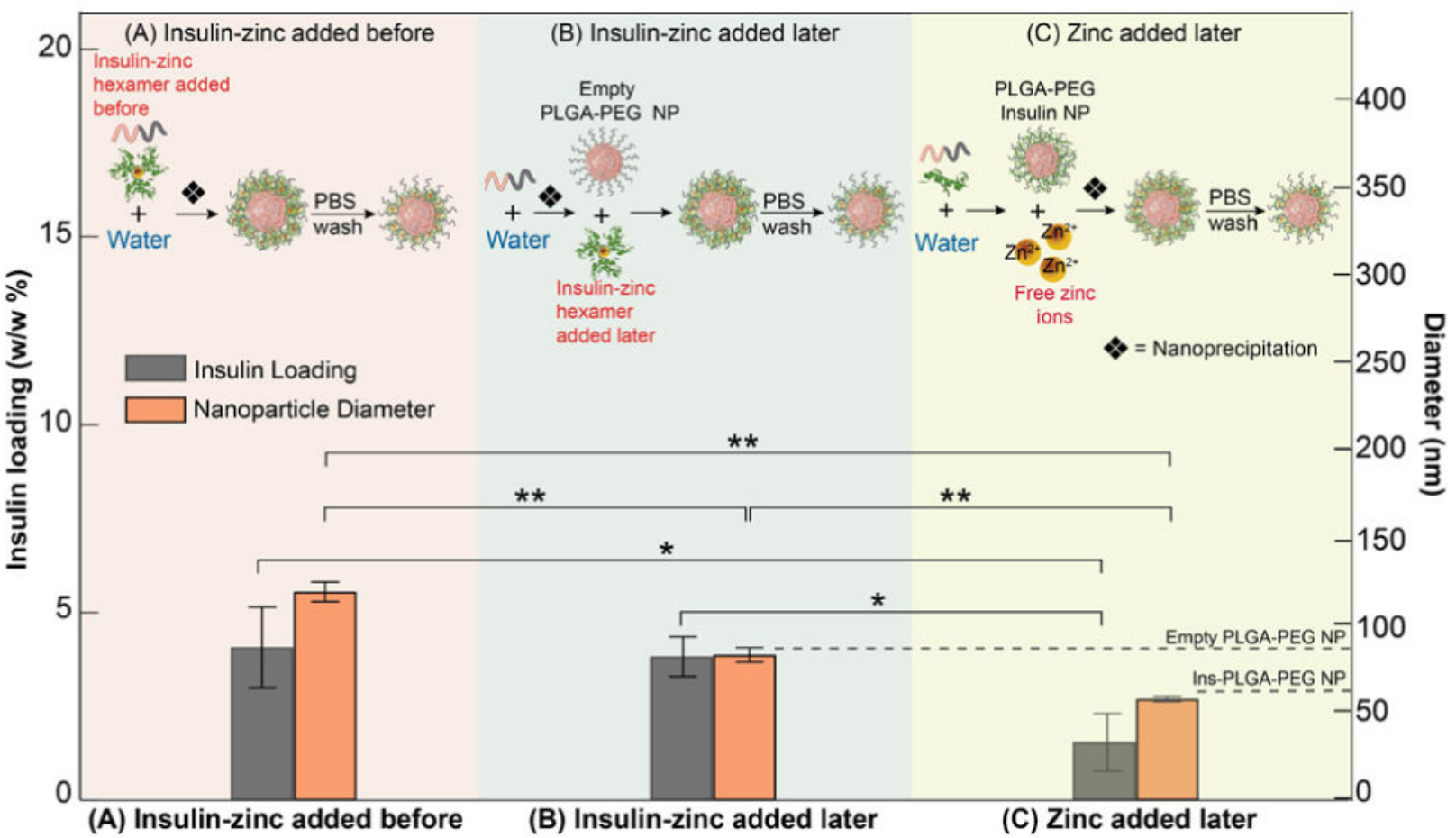

Figure 4. Effect of adding zinc ions at different stages of nanoprecipitation on Ins-Zn-NPs

(A) Schematic showing the synthesis of Ins-Zn-NPs by adding Ins-Zn hexamers premixed with PLGA-PEG molecules in DMSO before nanoprecipitation (top). This forms NPs with high insulin loading (4.07\%) and large NP diameter (127.6 nm). (B) Schematic showing the synthesis of Ins-Zn-NPs by adding Ins-Zn hexamers to empty PLGA-PEG NPs after nanoprecipitation (top). This forms NPs with high insulin loading (3.82\%) and small NP diameter $(78.7 \mathrm{~nm})$. (C) Schematic showing the synthesis of Ins-Zn-NPs by adding zinc ions to preformed Ins-NPs after nanoprecipitation (top). This forms NPs with lower insulin loading $(1.55 \%)$ and smaller NP diameter $(57.9 \mathrm{~nm})$. Dashed lines indicate sizes of empty PLGA-PEG and Ins-NPs for reference. Error bars represent \pm s.d. For insulin loading $n=3$ and $p_{A B}=0.74, p_{A C}=0.034, p_{B C}=0.016$. For NP diameter $n=3$ and $p_{A B}=0.0004, p_{A C}=$ $0.0001, p_{B C}=0.005$. 

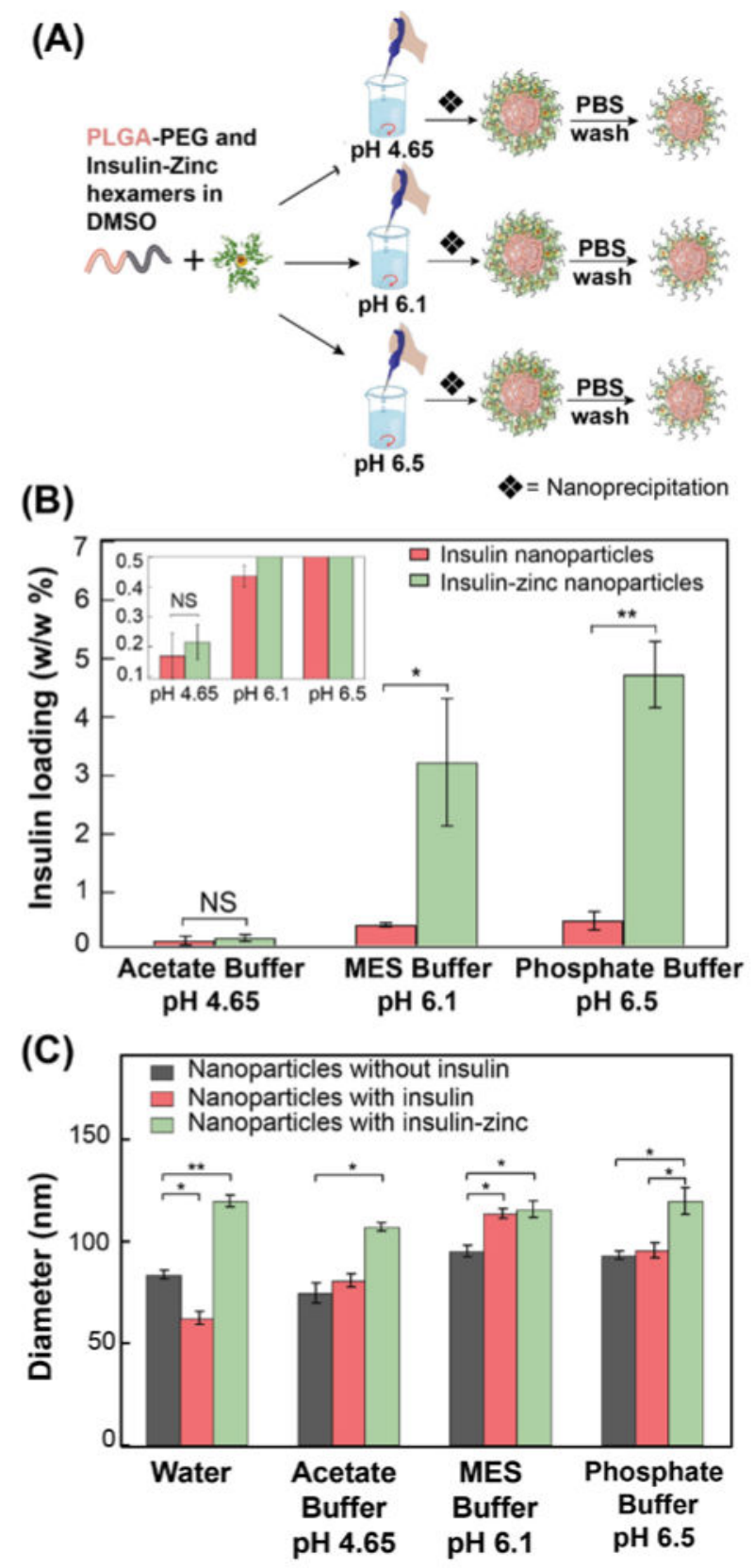

Figure 5. Effect of pH on Ins-Zn-NP and Ins-NPs

(A) Schematic showing the synthesis of Ins-Zn-NPs in different buffers ( $\mathrm{pH} 4.65, \mathrm{pH} 6.1$, and $\mathrm{pH}$ 6.5). (B) Insulin loading of Ins-Zn-NPs and Ins-NPs that were synthesized in buffers with different $\mathrm{pH}$. Insulin loading increased with increasing $\mathrm{pH}$. (C) Average diameter of empty PLGA-PEG NPs, Ins-NPs, and Ins-Zn-NPs synthesized in different buffers. 
(A)

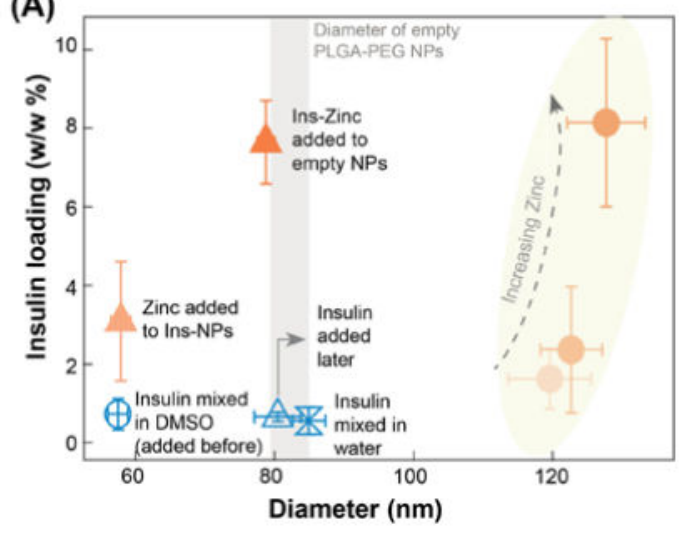

Insulin mixed in DMSO/ Insulin added before nanoprecipitation $\nabla$ Insulin mixed in water

$\triangle$ Insulin added to empty PLGA-PEG NPS after nanoprecipitation Ins-Zn-NPs (Ins: Zn::1:3)

Ins-Zn-NPs (Ins:Zn::1:6)

Ins-Zn added to empty PLGA-PEG NPs after nanoprecipitation(1:9)

A $\mathrm{Zn}$ ions added to Ins-NPs after nanoprecipitation(1:9)
(B)

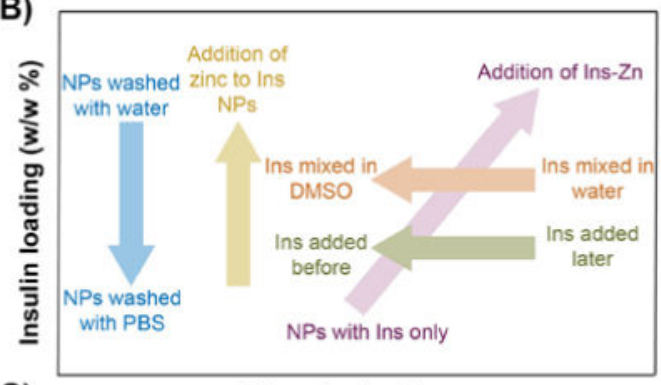

(C)

Diameter $(\mathrm{nm})$

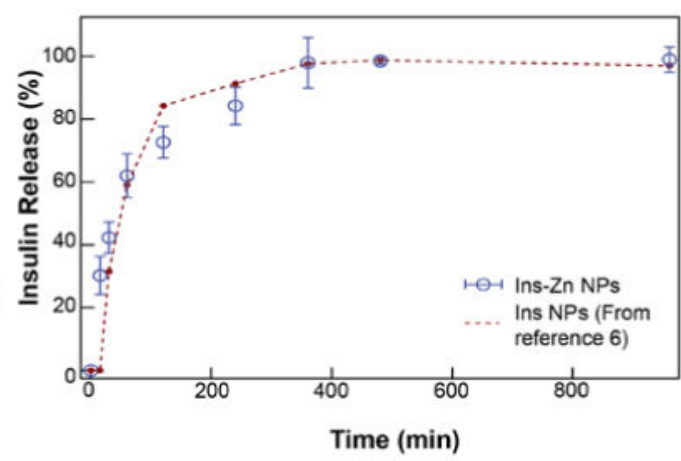

Figure 6. Compilation of the effect of NP synthesis parameters on NP properties (A) Compilation of the NPs studied for the purpose of understanding the factors that affect insulin loading and NP size in Ins-NPs and Ins-Zn-NPs. The symbols in blue contain only PLGA-PEG and insulin while the orange symbols represent NPs that contain PLGA-PEG, insulin, and zinc. The size of empty PLGA-PEG NPs has been represented by a grey vertical bar, whose width represents the standard deviation. Ins-NPs (blue) have low loading and small size. Contrary to these NPs, Ins-Zn-NPs formed by including zinc during nanoprecipitation (orange circles) have higher insulin loading, but their diameter is larger. NPs synthesized by adding Zn ions to preformed Ins-NPs (light triangle) show smaller size and moderate loading. The optimal NPs are Ins-Zn-NPs (dark triangle) formed by adding Ins-Zn hexamers to pre-formed NPs combine high loading (3.82\%) and small size (78.7 nm diameter). (B) Qualitative summary of how different synthesis parameters affect insulin loading and size. Insulin release. (C) Release of insulin from the optimal NPs (Ins-Zn-NPs with insulin loading $3.82 \%$, NP size $78.7 \mathrm{~nm}$ ) is comparable with the previously reported release of insulin from PLA-PEG NPs 6 . 